Cahiers $d u$ MONDE RUSSE

\section{Cahiers du monde russe}

Russie - Empire russe - Union soviétique et États indépendants

$52 / 4 \mid 2011$

Varia

\title{
Jean-Paul Bronckart, Cristian Bota, Bakhtine démasqué
}

\section{Serge Zenkine}

\section{(2) OpenEdition \\ Journals}

Édition électronique

URL : http://journals.openedition.org/monderusse/7509

DOI : 10.4000/monderusse.7509

ISSN : $1777-5388$

Éditeur

Éditions de l'EHESS

\section{Édition imprimée}

Date de publication : 20 décembre 2011

Pagination : 845-853

ISBN : 978-2-7132-2353-2

ISSN : $1252-6576$

\section{Référence électronique}

Serge Zenkine, « Jean-Paul Bronckart, Cristian Bota, Bakhtine démasqué », Cahiers du monde russe [En ligne], 52/4 | 2011, mis en ligne le 12 septembre 2013, Consulté le 21 septembre 2020. URL : http:// journals.openedition.org/monderusse/7509; DOI : https://doi.org/10.4000/monderusse.7509

Ce document a été généré automatiquement le 21 septembre 2020.

(c) École des hautes études en sciences sociales 


\title{
Jean-Paul Bronckart, Cristian Bota, Bakhtine démasqué
}

\author{
Serge Zenkine
}

\section{RÉFÉRENCE}

Jean-Paul BRONCKART, Cristian BOTA, Bakhtine démasqué : Histoire d'un menteur, d'une escroquerie et d'un délire collectif. Genève : Droz, 2011, 629 p.

1 C'est la rançon de la gloire : à force d'être vénérés et étudiés, certains auteurs célèbres se trouvent soumis à une critique textuelle si méticuleuse qu'elle finit par nier, sinon leur existence physique, du moins l'unité de leur œuvre et l'appartenance de ses parties à une seule et même personne. Les philologues ont longtemps débattu la « question homérique »; aujourd'hui, la même mésaventure arrive à Mihail Bahtin.

2 On connaît l'histoire du problème : vers 1970, quelques années après la redécouverte de l'œuvre du théoricien russe, il a été publiquement déclaré qu'en dehors des textes signés par lui (Dostoevskij, Rabelais et d'autres), il avait aussi écrit plusieurs livres et articles publiés dans les années 1920-1930 sous les noms de ses amis, principalement Valentin Vološinov et Pavel Medvedev. Après avoir été quasi dominante pendant une certaine période, cette opinion aujourd'hui, par un retour du pendule, voit diminuer le nombre de ses partisans : les «textes disputés » sont réédités et commentés comme des ouvrages de leurs signataires respectifs et ne figurent pas dans les œuvres complètes de Bahtin.

Deux critiques genevois, Jean-Paul Bronckart et Cristian Bota, ont fait un pas de plus : ils ont publié une longue enquête visant à déposséder Bahtin d'une partie même de son œuvre reconnue. Selon eux, non seulement il n'aurait aucun rapport aux ouvrages de Vološinov et Medvedev (« l'affaire des textes disputés est définitivement close »- p. 585), mais la première version de son livre sur Dostoevskij, parue en 1929, serait due pour une grande partie... à Vološinov, éventuellement aidé par Medvedev. Ce n'est pas Bahtin qui aurait prêté des textes à ses amis mais l'inverse. Quant à la légende de 
"l'omni-paternité bakhtinienne", attribuant à celui-ci des ouvrages de Vološinov et Medvedev, elle aurait été "conçue et construite dans le courant des années 60 " (p. 590), longtemps après la disparition des vrais auteurs; ils s'agirait d'un mensonge délibéré de Bahtin plagiaire ${ }^{1}$ et d'une escroquerie ourdie par ses " promoteurs » russes Vjačeslav Vs. Ivanov, Vadim Kožinov et Sergej Bočarov, «qui continuaient de gérer la lucrative diffusion internationale de l'œuvre reconstituée du maître » (p. 237). En plus, Kožinov et Bočarov auraient été "manifestement co-auteurs» (p. 272) de la seconde version du Dostoevskij (1963), Bahtin étant incapable de rédiger tout seul quelque ouvrage que ce soit et ne comprenant pas lui-même le sens de «son » livre qui ne l'était pas en réalité. Enfin, pour une cause inconnue, l'escroquerie aurait été dans la suite soutenue par nombre d'exégètes de différents pays, s'efforçant de rationaliser la légende de «l'omni-paternité bakhtinienne » et se livrant ainsi à un véritable délire interprétatif.

4 Cette critique anti-bakhtinienne, dont la violence se traduit par le ton souvent pamphlétaire de J.-P. Bronckart et C. Bota, n'est pas entièrement inédite, sauf peut-être en France. Ainsi, les légataires de Bahtin, à savoir Bočarov et Kožinov (mort en 2001), ont déjà été mis en cause (sans preuves) pour s'être approprié les droits d'auteur de Vološinov et Medvedev, par le fils de ce dernier Jurij Medvedev en $1995^{2}$. Et l'hypothèse de l'auctorialité collective du Dostoevskij de 1929 a été elle aussi évoquée en 2005, sans préjudice de Bahtin, par le linguiste russe Vladimir Alpatov ${ }^{3}$. Il est vrai que jusqu'à présent personne n'a développé ces conjectures d'une manière aussi circonstanciée, à longueur d'un livre comme celui de J.-P. Bronckart et C. Bota. Ce sont donc les détails de l'argumentation, les preuves alléguées qu'il s'agit d'examiner pour évaluer leur travail. Cette vérification nécessiterait un article beaucoup plus long que celui-ci : on se bornera à quelques aperçus généraux illustrés de quelques exemples.

5 J.-P. Bronckart et C. Bota n'apportent pas de nouveaux éléments au dossier. Ils ne révèlent pas de faits ni de textes inconnus, ils n'interrogent pas des documents qui ne l'aient déjà été. Tout en taxant la plupart des chercheurs qui les ont précédés d'avoir participé plus ou moins au "délire interprétatif», ils ne se servent pas moins uniquement des matériaux factuels et textuels rassemblés par ces mêmes chercheurs. Or, symptomatiquement, sauf quelques mentions insignifiantes, ils ne font aucun usage réel des richissimes commentaires contenus dans les Euvres complètes de Bahtin en sept volumes (Moscou, 1997-2011), une édition de référence pour tous ceux qui s'occupent de cet auteur.

6 Cette omission est d'ordre plus général: s'ils ont inclus dans leur imposante bibliographie quelques publications russes, J.-P. Bronckart et C. Bota n'en citent jamais les textes; toutes leurs citations proviennent des sources en d'autres langues. Il est permis de supposer que les deux auteurs ne maîtrisent pas la langue russe, ce qui compromettrait la validité de leur analyse: imaginez un helléniste qui prétende trancher la question homérique sans connaître le grec! En effet, obligés de faire confiance aux traductions des textes russes, qui ne sont pas toujours fidèles, ils s'aventurent parfois dans des interprétations hasardeuses. Un exemple : parmi les propos de Bahtin enregistrés par Victor Duvakin en 1973, la réplique commençant par «Non, que dites-vous!...» (p.267) et occupant sept lignes, comporte trois graves erreurs de traduction (il s'agit d'une traduction seconde, effectuée par J.-P. Bronckart et $\mathrm{C}$. Bota à partir d'une version italienne) ${ }^{4}$. D'abord, Bahtin caractérise Vadim Kožinov comme « une personne absolument sans scrupules»-l'original dit «une personne 
absolument sans peur [besstrašnyj] ». Ensuite, le mot de Bahtin minusnik, par lequel il se désigne lui-même, est traduit comme " moins que rien » - en fait, il s'agit d'un terme de jargon administratif de l'époque stalinienne, signifiant un déporté, interdit de résidence dans un certain nombre de grandes villes (on disait minus desjat', minus dvadcat' - «moins dix, moins vingt »). Enfin, et c'est le plus curieux, suit une phrase de Bahtin d'apparence étrange et suspecte : «Ce livre de lui avait été oublié, le Dostoïevski je veux dire...»; en effet, qui est ce « lui »- serait-ce Vološinov ? Voici le commentaire de J.-P. Bronckart et C.Bota: "On remarquera l'hésitation de Bahtin concernant l'ouvrage sur Dostoevskij, qu'il a peine manifestement à appeler "mon livre" » (p. 268). Or, l'original est fort simple : « Knižku etu zabyli - "Dostoevskogo"... » [Ce petit livre a été oublié, le «Dostoevskij... »] - et ne présente aucune hésitation ni anomalie linguistique, les déterminatifs possessifs étant moins fréquents et moins obligatoires en russe qu'en français. Sa lecture suspicieuse tient à un pur malentendu.

7 Il y a des malentendus autrement plus graves. J.-P. Bronckart et C. Bota semblent ignorer tout le contexte historique, biographique et institutionnel dans lequel ont été produits les dits et les écrits de Bahtin et de ses amis. Ils confrontent, à la manière d'un enquêteur policier, les propos souvent évasifs de Bahtin au sujet des " textes disputés ", sans faire le moindre cas des changements du climat politique, des interlocuteurs, de la situation matérielle, de l'âge et de l'état de santé qui ont pu l'inciter tantôt à l'ouverture et tantôt à la circonspection (que ce vieux minusnik, victime de persécutions politiques, avait dû bien apprendre et qu'il montrait à maint propos, non seulement en ce qui concerne les "textes disputés »). Ils chargent de l'accusation sordide d' escroquerie les trois «promoteurs» de Bahtin comme si c'étaient des inconnus, des hommes obscurs, sans s'enquérir (ce que ferait un bon policier) de leur réputation morale et professionnelle - pourtant au moins l'un d'eux, le sémioticien Vjačeslav Vsevolodovič Ivanov ${ }^{5}$, jouit d'une renommée mondiale; ils amalgament les trois personnages en une association de malfaiteurs, une «troïka moscovite» (p. 245), tout en ignorant d'importantes divergences littéraires et idéologiques entre certains d'eux. Quant au contexte historico-social, ils s'imaginent par exemple que les purges staliniennes qui ont emporté des millions de vies autour de 1937, frappaient « surtout voire exclusivement - les membres du parti et les enseignants marxistes» (p. 33); ils n'admettent pas, d'une façon fort naïve, qu'une petite université provinciale de Saransk (initialement un "institut pédagogique " formant des maîtres d'école), où Bahtin a enseigné dans les années 1930-1950, ait pu être dépourvue d'une bonne bibliothèque de recherche (ou de librairie - p. 317$)^{6}$; ils suggèrent à plusieurs reprises que Bahtin ne pouvait pas être un leader intellectuel par rapport à ses amis Medvedev et Vološinov parce que, à la différence d'eux, il n'avait pas de diplôme universitaire ${ }^{7}$ et n'était pas un " chercheur chevronné » (p. 293), - probablement sans savoir que la chose n'était pas exceptionnelle en Russie révolutionnaire, où par exemple un opposant de Bahtin en théorie littéraire, le formaliste Victor Šklovskij, a bien pu fonder et animer la célèbre Société d'étude du langage poétique (OPOJAZ) sans avoir terminé ses études supérieures et en gagnant sa vie comme écrivain, scénariste, militaire et parfois même conspirateur clandestin.

8 Des documents importants pour la biographie de Bahtin et la question des «textes disputés ", qui ne sont pas tous traduits du russe, échappent à l'attention de J.P. Bronckart et C. Bota. Ceux-ci laissent entendre que les «promoteurs» de Bahtin, après avoir proclamé l'appartenance à cet auteur des "textes disputés ", n'ont jamais cité leurs sources - en réalité, ils l'ont fait en 1995 : V.Vs. Ivanov a précisé qu'il avait 
appris la chose (sur Marxisme et philosophie du langage de Vološinov) dès 1956 de l'académicien linguiste Victor Vinogradov ${ }^{8}$; et Vadim Kožinov de son côté a confirmé tenir l'information (sur La Méthode formelle en littérature de Medvedev) du même Vinogradov, et aussi des éminents critiques littéraires Naum Berkovskij et Victor Šklovski. Peut-être J.-P. Bronckart et C. Bota refusent-ils toute confiance à ces gens-là ; mais, au lieu de critiquer leurs témoignages, ils les passent sous silence. Ils font de même avec les mémoires inédits de l'helléniste Olga Frejdenberg qui avait connu Vološinov et affirmait elle aussi que son "livre linguistique " avait été écrit par une autre personne ${ }^{10}$. O. Frejdenberg étant morte en 1955, sa seule phrase suffirait à invalider la thèse selon laquelle " après les déclarations de paternité de Bahtin et leur diffusion par Ivanov, aucun témoignage émanant de cette période [entre 1925 et 1970] et confirmant la chose n'a jamais été produit » (p. 82) - au contraire, tout porte à croire qu'il s'agit d'une rumeur qui a circulé pendant la période en question dans les milieux académiques et littéraires et qui a été consignée au moins dans un document d'époque. Qu'elle soit vraie, ou fausse, ou en partie vraie et en partie fausse, elle a bien existé, Bahtin et ses collègues ne l'ont pas inventée dans les années 60 ; du coup, toute l'hypothèse de leur « escroquerie » s'écroule.

Dans l'argumentation mise en œuvre par J.-P. Bronckart et C. Bota, il faut signaler l'emploi fréquent de lectures abusives, de déductions tendancieuses et de glissements logiques. Bahtin avoue à Duvakin: «La manière dont j'aurais pu écrire [le livre sur Dostoevskij] aurait été bien différente de ce qu'elle est»; les deux critiques de commenter qu' " en hypothèse forte, cela peut signifier que Bahtin n'a pas écrit ce livre ", et qu'ils préfèrent cette hypothèse-là à "l'hypothèse faible ", pourtant d'évidence conforme à la suite de la conversation, selon laquelle « il l'a écrit, mais pas comme il le souhaitait » (p. 254). En 1928, Boris Pasternak écrit à Pavel Medvedev, après avoir lu sa Méthode formelle en littérature, qu'il ne savait cet auteur-là être « un tel philosophe »; on cite parfois cette appréciation pour montrer le statut exceptionnel de l'ouvrage (censé appartenir en réalité à Bahtin) parmi les autres écrits de son signataire ; non, répliquent J.-P. Bronckart et C. Bota, ce n'est qu'une louange banale «je ne vous croyais pas aussi brillant » (p. 67); si fait, répondra-t-on, car le poète ne parle pas de degrés de brio mais, avec plus de précision et de tact, d'une différence de nature entre deux discours, la critique littéraire des ouvrages précédents de Medvedev et la philosophie dont s'occupait Bahtin. À Sergej Bočarov, Bahtin dit qu'il a écrit les livres «disputés" "du début à la fin» (p. 244); et à Vadim Kožinov, que Pavel Medvedev y a introduit quand même "certaines adjonctions [...] pas très heureuses " (p. 246). De leur regard inquisiteur, J.-P. Bronckart et C. Bota détectent une "totale contradiction» (p. 247) entre ces deux déclarations: sans doute un indice des mensonges de Bahtin que les «complices de ces mensonges» (p. 271), mal concertés entre eux, n'ont pas su dissimuler... Mais, en bonne logique, pourquoi le fait d'avoir rédigé un texte du début à la fin exclut-il qu'en ce faisant on a pu s'inspirer des idées des autres ou qu'on a pu dans la suite laisser aux autres le soin de le remanier, voire de le compléter avec des interpolations?

Bahtin a-t-il donc menti? Il lui arrivait bien de fournir des informations fausses sur sa biographie (son origine sociale, ses études); mais sur le fond de la question des «textes disputés " il ne se contredit jamais ni ne contredit directement les faits dont nous disposons. J.-P. Bronckart et C. Bota n'ont pas su prouver le contraire : ce qui varie dans ses propos, ce sont les motifs, les explications des actes, la plupart du temps conciliables entre elles; mais il ne dit jamais formellement qu'il n'a pas écrit les textes 
problématiques, ni qu'il les a écrits tout seul, sans aucune participation des autres. Sa position est toujours nuancée, entre les deux : tantôt il s'avoue l'auteur principal des textes, nonobstant quelques retouches faites par ses amis, tantôt il se borne à affirmer « une conception commune du langage et de l'œuvre verbale » (p. 241) que ces textes-là partagent avec ses propres écrits de l'époque et les uns avec les autres. Le dernier fait semble incontestable, et il est indirectement reconnu par J.-P. Bronckart et C. Bota : « ... l'approche de Medvedev est absolument identique à celle développée par Vološinov " (p. 493). Seulement, puisque en l'occurrence il s'agit de rapports de Medvedev et Vološinov entre eux et non avec Bahtin, ils ne crient pas au plagiat ni à la substitution d'auteur...

11 En revanche, la même communauté d'idées sera utilisée pour désavouer l'auctorialité du Dostoevskij. Selon J.-P. Bronckart et C. Bota, les amis de Bahtin se seraient employés à transformer ses fragments confus d'inspiration religieuse en un ouvrage novateur en sciences humaines, à la suite des recherches propres de Vološinov; plus simplement, "Voloshinov a décidé de publier sous le nom de [Bahtin] une part du travail qu'il conduisait alors» (p.555). La publication du livre serait destinée à affermir la réputation professionnelle de Bahtin et à adoucir son sort, car en 1929 il allait être jugé pour ses activités dans les cercles religieux de Leningrad. L'hypothèse est ingénieuse ; l'ennui, c'est qu'elle ne rentre pas dans les cadres chronologiques. Ses auteurs laissent entendre (p. 271-272 et ailleurs) que Bahtin ne pouvait pas rédiger et éditer son livre, parce qu'en décembre 1928 il avait été arrêté par la police politique ; or, en réalité il n'a passé que quelques jours à la maison d'arrêt, après quoi, avant de partir en exil en février 1930, il a demeuré à Leningrad en résidence surveillée, ce qui ne devait pas l'empêcher de s'occuper de l'édition de son ouvrage ${ }^{11}$. Il est donc faux que le processus de cette édition "a manifestement été géré par d'autres en raison de l'incarcération puis de la déportation du signataire» (p. 51) ${ }^{12}$. Ce dernier a d'ailleurs déclaré avoir terminé son livre avant l'arrestation, en 1928 (p. 267), et son témoignage est confirmé par des documents éditoriaux retrouvés (en 1928, Bahtin avait signé un contrat, portant le 15 octobre comme la dernière date de livraison du manuscrit) et par la date du premier compte-rendu du Dostoevskij publié dans la presse le 10 juin 1929. Ces derniers faits, les deux critiques genevois ne les discutent pas - ils les ignorent, comme ils ignorent à peu près tout l'appareil critique des Euvres complètes où ces faits ont été rapportés ${ }^{13}$. Or, la composition, les épreuves et l'impression demandant au moins cinq à six mois ${ }^{14}$, pour qu'un livre ait pu paraître début juin 1929 son manuscrit devait bien être livré à l'éditeur avant fin 1928. Après l'arrestation de Bahtin, Vološinov ou n'importe qui d'autre n'auraient pas eu le temps de réélaborer son texte, en eussent-ils eu l'intention.

Les arguments biographiques et factuels se révélant défectueux, qu'en est-il des autres arguments, tirés de l'analyse des textes théoriques? J.-P. Bronckart et C. Bota cherchent à montrer que, par la teneur de ses idées principales, le premier Dostoevskij est plus proche des ouvrages de Medvedev et Vološinov que des premiers écrits philosophiques de Bahtin (Pour une philosophie de l'acte, L'Auteur et le héros); peut-être, mais pourquoi expliquer cette proximité par l'hypothèse singulière que le Dostoevskij n'est pas l'œuvre de Bahtin, et non par le simple fait, que celui-ci a reconnu, d'un échange intellectuel intense entre les trois hommes, qui allait jusqu'à la mise en place d'une "conception commune" de la culture verbale? Que, dans le Dostoevskij, cette conception commune coexiste avec des restes des premières théories philosophiques de Bahtin, cela est assez naturel, et en principe on pourrait soutenir, ce que font J.P. Bronckart et C. Bota, qu'ils ne s'accordent pas toujours ensemble - mais cela ne suffit 
pas à conclure que le texte de l'ouvrage ait été rédigé par deux ou trois personnes différentes; il serait plus logique de supposer, comme on l'a déjà fait, que l'auteur a travaillé longtemps sur son texte, et que ce dernier se compose des parties datant de différentes périodes.

Il est vrai que, pour en arriver à la "conception commune ", Bahtin a dû faire une évolution prodigieusement rapide - d'une philosophie phénoménologique et religieuse vers des sciences humaines objectives et sociologisantes. Cependant son cas n'est pas unique : parmi ses amis proches, une évolution assez semblable se retrouve chez Lev Pumpjanskij et surtout Valentin Vološinov qui, quelques années avant de s'inspirer du "matérialisme dialectique» (p.582), avait dû être un Rose-Croix ${ }^{15}$; ce genre de conversion a été également fréquente dans l'intelligentsia européenne de l'époque, chez le mystique Walter Benjamin ou le phénoménologue Jean-Paul Sartre, pour ne citer que les plus célèbres. Les motifs de l'évolution ont pu varier : développement libre de la pensée, lectures et influences intellectuelles, pression idéologique (surtout en Russie soviétique), etc., - or, sans examiner ces possibilités, J.-P. Bronckart et C. Bota leur en préfèrent une autre: il n'y aurait pas eu d'évolution du tout mais une simple substitution de personnes, suivie d'un "scandaleux détournement d'auctorialité » (p. 271) ${ }^{16}$.

14 Pour donner de la vraisemblance à cette thèse, ils sont obligés d'exagérer l'incompatibilité des deux programmes de recherche entre lesquels Bahtin devait évoluer : ces deux approches, disent-ils, seraient «radicalement disjointes et largement antagonistes » (p. 507). En accentuant les tendances religieuses de la première esthétique bakhtinienne - qu'ils ne cessent de vilipender comme une «idéologie réactionnaire » (p. 393), une " perspective régressive et destructrice» (p. 408), " une idéologie radicalement réactionnaire» ( $i b i d$.$) , un «tissu de considérations ultra-phénoménologiques et$ bondieusardes» (p. 515) - ils croient y déceler « un monologisme radical» (p. 410) en contradiction avec le principe dialogique du Dostoevskij et des écrits de Vološinov. Il ne s'aperçoivent pas que la notion de monologisme, définie dans le Dostoevskij comme la domination d'un discours, est inapplicable aux ouvrages où le problème du langage n'est pas encore posé, tandis que la situation de dialogue y est anticipée par une analyse de la communication entre les hommes - et non seulement entre l'homme et Dieu - ne se fondant pas sur la domination mais sur l'amour.

15 Le même souci de creuser l'abîme entre les deux programmes amène J.-P. Bronckart et C. Bota à surestimer le « marxisme » des " textes disputés » de la fin des années 20 . En fait, qu'il soit sincère ou imposé du dehors (problème qui divise depuis longtemps les exégètes, les uns interprétant ces textes «à gauche " et les autres "à droite »), ce marxisme reste rudimentaire, ce qu'a montré par exemple tout récemment Patrick Sériot à propos de la philosophie du langage de Vološinov ${ }^{17}$. C'est un marxisme sans dialectique, sans lutte de classes ni révolution, sans idées de praxis, de travail ni d'idéologie (le dernier terme, fréquent chez Vološinov, y a un sens qui ne correspond que peu à L'Idéologie allemande), un marxisme qui se réduit au principe sommaire de « monisme matérialiste » (p. 417) ${ }^{18}$ et à un « interactionnisme social» (p. 464), privilégiant des solidarités et non des conflits sociaux entre les individus communicants. J.P. Bronckart et C. Bota, qui connaissent l'article de P. Sériot, ne tentent pas de démentir une seule de ces critiques. Or, réorienter les sciences du langage et de la littérature vers une étude de l'interaction humaine et mettre cet aspect interactionniste du langage audessus de son aspect cognitif - un geste théorique important dont on peut à juste titre 
créditer les auteurs des «textes disputés » - tout cela n'implique pas pour autant leur " ancrage résolu dans le marxisme » (p. 415), loin s'en faut.

On pourrait discuter encore longtemps, mais les conclusions s'imposent. À vouloir prouver trop, J.-P. Bronckart et C. Bota n'ont prouvé rien, ils n'ont fait qu'embrouiller encore plus une question déjà compliquée. Le dossier des «textes disputés » n'est pas classé - et ne le sera peut-être jamais, à moins qu'on ne découvre quelques nouvelles pièces décisives; l'hypothèse d'auctorialité collective du Dostoevskij pourrait être plausible mais, privée de preuves convaincantes et assortie d'une chronologie improbable, elle reste gratuite; le procès des «mensonges" de Bahtin au sujet des textes censés constituer son œuvre doit se clore par un non-lieu, toujours faute de preuves et en vertu de la présomption d'innocence; le récit rocambolesque d'une " escroquerie » prétendument commise par ses collègues russes pourrait être qualifié de calomnie s'il ne s'expliquait plus simplement par une ignorance patente des faits. D'une manière générale, les graves lacunes documentaires, les traductions et lectures erronées, les déductions forcées et les distorsions logiques qui se multiplient dans l'ouvrage de J.-P. Bronckart et C. Bota ne peuvent que dévaloriser leurs analyses textuelles, souvent fort détaillées mais discréditées par leur visée tendancieuse.

L'œuvre de Mihail Bahtin est difficile. On y trouve bien des obscurités, des emprunts inavoués, des contradictions apparentes et réelles, des textes plus ou moins achevés et plus ou moins réussis; nous manquons d'éléments factuels pour démêler les apports que lui-même et ses amis et collègues ont pu faire, dans les années 1920, à quelques textes résultant de leur échange et de leur fonds commun d'idées. Certains bakhtinistes, il faut bien le dire, ont aggravé cette difficulté objective avec leur zèle excessif tendant à ériger un culte de Bahtin. Pour redresser la situation, on a besoin d'une critique compétente et patiente, qui ne cherche pas des solutions sensationnelles, qui ne réduit pas les complexités d'une pensée théorique à des plagiats et détournements des droits d'auteur, et qui ne traite pas de menteurs, d'escrocs et de délirants ceux qui disent le contraire d'elle. L'hétérogénéité du corpus bakhtinien est un bon problème - c'est dommage s'il tombe entre de mauvaises mains.

\section{NOTES}

1. «Ses propres écrits tardifs relèvent manifestement du plagiat » (p. 274, ici et dans la suite, les gras et les italiques dans les citations appartiennent aux auteurs du livre).

2. Voir Ju.P. Medvedev, « Pis'mo v redakciju zhurnala...” [Lettre à la rédaction du journal], Dialog. Karnaval. Hronotop, $\mathrm{n}^{\circ} 4,1995$, p. 154. Ce document précurseur est absent de la bibliographie de J.P. Bronckart et C. Bota.

3. Voir Vladimir ALPATov, Vološinov, Bahtin i lingvistika [Vološinov, Bahtin et la linguistique], M. : Jazyki slavjanskih kul'tur, 2005, p. 117. Cet ouvrage figure dans la bibliographie de J.-P. Bronckart et $C$. Bota, sans que sa priorité sur ce sujet-là soit signalée.

4. Cf. l'original russe: Besedy V.D.Duvakina s M.M. Bahtinym [Entretiens de V.D. Duvakin avec M.M. Bahtin], M. : Progress, 1996, p. 217-218. 
5. L'index de l'ouvrage de J.-P. Bronckart et C. Bota (p. 618, référence à la page 266) ne fait pas de distinction entre lui et son homonyme, le poète Vjačeslav Ivanovič Ivanov (1866-1949). Le même index (p.621), et aussi le texte du livre (p. 32), attribuent à l'écrivain Léon (Lev Nikolaevič) Tolstoj des initiales fantasques « N.J.».

6. Les deux auteurs s'en prennent à une phrase de Brian Poole qu'ils traduisent comme « Bakhtine a rarement vécu près d'une bonne librairie» (p. 316). L'original anglais (The South Atlantic Quarterly, 97, 1998, p. 568) dit library - «bibliothèque ».

7. La question des études que Bahtin a faites en sa jeunesse n'est pas bien éclaircie. On suppose qu'il a dû suivre les cours universitaires sans être inscrit (voir N.L. vasil'ev, «Kommentarii k kommentarijam» [Commentaires des commentaires], Dialog. Karnaval. Hronotop, $\mathrm{n}^{\circ}$ 4, 1995, p. 160).

8. Voir V.Vs. Ivanov, «Ob avtorstve knig V.N. Vološinova i P.N. Medvedeva » (Sur l'auctorialité des livres de V.N. Vološinov et P.N. Medvedev), Dialog. Karnaval. Hronotop, n 4, 1995, p. 134.

9. Voir V. Kožinov, « Kniga, vokrug kotoroj ne umolkajut spory » [Le livre dont on ne cesse de discuter], ibid., p. 140.

10. Cité par Nina Perlina dès en 1988. Voir une publication commentée, avec une reproduction photographique du manuscrit: Nina Braginskaja, «Meždu svideteljami i sud'jami [Entre les témoins et les juges]», dans M.M. Bahtin v Saranske [M.M. Bahtin à Saransk], Saransk, 2006, p. 39-60, http://ivgi.rsuh.ru/article.html?id=207419.

11. Le fait, rapporté par Bočarov (p. 259) et contesté par J.-P. Bronckart et C. Bota comme étant "en contradiction avec d'autres données historiques»(p.260), est confirmé par le dossier judiciaire de Bahtin. Voir S.S. Konkin et L.S. Konkina, Mihail Bahtin, Saransk, 1993, p. 185-186.

12. Il est également faux qu'en rééditant le Dostoevskij en 1963, Bahtin n'ait pas pu «accomplir lui-même ce travail et, comme il l'indiquait notamment dans ses entretiens avec Duvakin, c'est en réalité Kožinov qui [ait] géré l'entreprise de réélaboration» (p.519), que «le remaniement effectif [ait été] géré pour l'essentiel par Kožinov ... » (p. 539 ; cf. la même affirmation p. 272, 590 et ailleurs). En vérité, comme Bahtin l'explique à Duvakin (voir l'édition russe citée, p. 218), Kožinov a obtenu que le livre soit publié, il l'a " promu », " propulsé » (prodvinul, prochibal) à une maison d'édition de Moscou; mais c'est Bahtin lui-même qui a effectué tout le travail de retouches et d'addition, y compris la rédaction d'un chapitre supplémentaire sur le problème du genre. En témoignent par exemple ses manuscrits du second Dostoevskij, publiés dans le volume 6 des Euvres complètes en 2002 et ignorés par J.-P. Bronckart et C. Bota, qui ne semblent connaître de seconde, voire de troisième main, d'après Kozhinov cité en anglais par N. Rzhevsky - qu'un court aide-mémoire rédigé par Bahtin au début de ce travail, en 1961.

13. Voir Mihail Bahtin, Sobranie sočinenij [Guvres complètes], t.2, M.: Russkie slovari, 2000, p. $432,473$.

14. Tel a dû être par exemple le délai d'édition du livre de Yuri Tynjanov Les Archaïstes et les novateurs, paru chez le même éditeur à la même époque, en janvier-février 1929.

15. Voir Patrick Sériot, « Préface », dans Valentin Nikolaevič vološinov, Marxisme et philosophie du langage, Limoges : Lambert-Lucas, 2010, p. 52-54.

16. Un autre cas de non-examen des arguments alternatifs : en 2001, Brian Poole, en étudiant les références implicites des premiers écrits de Bahtin, a cru démontrer qu'ils avaient été rédigés vers 1927, c'est-à-dire plus tard qu'on ne le pensait et au même moment que les premiers « textes disputés ». Si cette nouvelle datation était correcte, elle réduirait à zéro le temps de l'évolution de Bahtin dans les années 20, et rendrait celle-là peu probable. Or, dès en 2004, Nikolaj Nikolaev et Vadim Ljapunov, les annotateurs russe et américain du volume 1 des Euvres complètes de Bahtin, ont donné une critique sérieuse, avec un important matériau factuel, de l'hypothèse de Poole. Tout en connaissant de seconde main (voir leur note de la page 513, renvoyant à une publication suisse de 2005) l'existence de cette critique, J.-P. Bronckart et C. Bota ne se prononcent point sur sa validité, et reprennent telles quelles les thèses de Poole (voir p. 293, 
512-513 et ailleurs). Encore une fois, on en vient à supposer qu'ils n'ont pas consulté de visu les œuvres complètes de l'auteur étudié : une chose déconcertante, c'est le moins qu'on puisse dire. 17. Voir Patrick sériot, "Préface", p. 54 sq. L'analyse de Sériot pèse d'autant plus que ce chercheur écarte l'hypothèse de "l'omni-paternité bakhtinienne» et considère Marxisme et philosophie du langage comme une œuvre de Vološinov.

18. En posant ce principe, J.-P. Bronckart et C. Bota le font remonter (p. 417) à deux textes : L'Éthique de Spinoza et l'ouvrage polémique de Lenin Matérialisme et empiriocriticisme, qui ne semblent guère les meilleures expositions des idées de Marx. 